\title{
Influencia de la atmósfera de sinterización en la estabilidad térmica de cerámicas de hidroxiapatita reforzadas con circona
}

\author{
* J. A. DELGADO GARCíA-MENOCAL, L. MOREJÓN ALONSO ** S. MARTÍNEZ MANENT \\ *** J. RODRÍGUEZ VIEJO, M. T. MORA AZNAR \\ * Centro de Biomateriales, Universidad de La Habana, 10400 Ciudad Habana, Cuba \\ ** Departamento de Cristalografía, Mineralogía y Depósitos Minerales, Facultad de Geología, Universidad de Barcelona, 08028 Barcelona, España \\ *** Grupo de Física de Materiales I, Departamento de Física, Universidad Autónoma de Barcelona, 08193 Barcelona, España
}

\begin{abstract}
En este trabajo se estudia la influencia de la atmósfera de sinterización sobre la estabilidad térmica de cerámicas de hidroxiapatita (HA) reforzadas con partículas de circona parcialmente estabilizada con magnesia (Mg-PSZ). Los cuerpos verdes fueron obtenidos por prensado uniaxial y las sinterizaciones se realizaron en aire y en oxígeno húmedo. El estudio por difracción de rayos- $X$ y espectroscopía infrarroja permitió comprobar que la atmósfera húmeda evita la descomposición de la hidroxiapatita, todo lo contrario a lo que sucede en aire donde es evidente la presencia del zirconato cálcico (CZ) en la interfaz entre la partícula de circona y la matriz de HA. La caracterización microestructural de los materiales por Microscopía Electrónica de Barrido permitió apreciar el desarrollo de tamaño de grano de los materiales sinterizados. El estudio del comportamiento mecánico mediante ensayos de indentación reveló un aumento de la tenacidad a la fractura en el caso de los materiales que presentaban Mg-PSZ con respecto a la HA pura.
\end{abstract}

Palabras clave: hidroxiapatita, circona, cerámica, estabilidad térmica, propiedades mecánicas

Influence of the sintering environment in the thermal stability of hydroxyapatite ceramics reinforced with zirconia.

In this work the influence of the sintering environment in the thermal stability of the hydroxyapatite ceramics (HA) reforced with magnesia-partially-stabilized-zirconia (Mg-PSZ) particles is studied. The green bodies were prepared by uniaxial pressing and the sintering as carried out both in air and humid oxygen. The powder X-ray diffraction patterns and infrared spectroscopy spectra showed that the humid environment sintering avoids the decomposition of HA. On the contrary, when the sintering is done in air the calcium zirconate $(\mathrm{CZ})$ appears owing to reaction between the zirconia and calcium oxide released due to transformation of HA. The grain growth in the sintered materials was confirmed by Scanning Electronic Microscopy. The mechanical behavior was studied by indentation methods and the results obtained revealed an increase in the fracture toughness of the materials reinforced with $\mathrm{Mg}$-PSZ respect to $\mathrm{HA}$.

Key words: hydroxyapatite, zirconia, ceramics, thermal stability, mechanical properties

\section{INTRODUCCIÓN}

Las cerámicas de HA constituyen uno de los biomateriales que más interés han despertado en los últimos años como material de implante óseo por la excelente biocompatibilidad con el tejido del hueso (1-3). Pero su aplicación en la cirugía reconstructiva ha estado limitada a zonas del organismo donde no existan esfuerzos mecánicos importantes debido a su baja resistencia a la fractura y a la fatiga (4). Entre las variantes que se han empleado para solucionar este problema, se encuentra la utilización de materiales compuestos a base de HA reforzados con circona parcialmente estabilizada (PSZ) con óxido de itrio (Y-PSZ) (5). Las excelentes propiedades mecánicas de la PSZ se atribuyen a la denominada transformación tenaz "toughening transformation" que tiene lugar en el material cuando la fase tetragonal metaestable se transforma en fase monoclínica por el efecto de esfuerzos mecánicos inducidos (6).

En materiales compuestos de HA-PSZ obtenidos por prensado uniaxial, la difusión del calcio hacia la PSZ en la sinterización provoca la descomposición de la estructura apatítica y la transformación de la PSZ en zirconato cálcico con la consi- guiente disminución de sus propiedades mecánicas (7). En este trabajo se ha empleado circona parcialmente estabilizada con magnesia (Mg-PSZ) como agente de refuerzo. Los materiales se prepararon por prensado uniaxial y las sinterizaciones se realizaron en aire y en atmósfera húmeda teniendo en cuenta la estabilidad a altas temperaturas de la HA en condiciones de humedad (8). Además de comprobar si la sinterización en atmósfera húmeda evitaba la descomposición de la HA, se realizó la caracterización microestructural y de composición de fases de los cerámicos así como la determinación de la tenacidad a la fractura mediante ensayos de indentación.

\section{PARTE EXPERIMENTAL}

\subsection{Preparación de los materiales}

La HA empleada en este trabajo se obtuvo por reacción entre una suspensión en agua de hidróxido de calcio (Merck) y una solución de ácido ortofosfórico (Merck). La Mg-PSZ fue obte- 
nida a partir de polvo de circona (Merck) y óxido de magnesio (Panreac) (3\% en peso), dicha mezcla se calcinó a $1450^{\circ} \mathrm{C}$ durante 4 horas a una velocidad de calentamiento de $120^{\circ} \mathrm{C} / \mathrm{h}$. y se enfrió a unos $300^{\circ} \mathrm{C} / \mathrm{h}$. El material obtenido se trituró hasta un tamaño de partícula de $7.7 \mu \mathrm{m}$ y se mezcló con el polvo de HA, previamente calcinada a $700^{\circ} \mathrm{C}$ durante dos horas, en una proporción de $20 \%$ en peso de Mg-PSZ empleando alcohol etílico para lograr una mayor homogeneidad en el material resultante. En las muestras preparadas para estudiar la composición química de la capa de difusión por microsonda electrónica, el tamaño de las partículas de circona fue de alrededor de $100 \mu \mathrm{m}$.

Los compactos se prepararon por prensado uniaxial, en discos de $6 \mathrm{~mm}$ de diámetro, con un rango de presiones entre los 175 y los $440 \mathrm{MPa}$. Las sinterizaciones se llevaron a cabo en un horno tubular a temperaturas de $1250^{\circ} \mathrm{C}$ y $1350^{\circ} \mathrm{C}$ por espacio de 4 horas a velocidades de calentamiento y enfriamiento de $120^{\circ} \mathrm{C} / \mathrm{h}$. La atmósfera húmeda se logró haciendo pasar oxígeno por agua destilada hirviendo, el gas saturado en vapor de agua se hizo circular por el interior del tubo del horno. La textura se puso de manifiesto mediante ataque químico durante $30 \mathrm{~s}$ con una mezcla de $\operatorname{EDTA}(0.2 \mathrm{M})$ y ácido láctico $(85 \%)$ en una proporción de 1:1.

\subsection{Técnicas de caracterización}

\subsubsection{ANALISIS QUÍMICO}

Para la caracterización química la HA se disolvió con ácido nítrico concentrado. La determinación del contenido de calcio y fósforo se realizó con un equipo de Plasma de Inducción Acoplado (ICP) Thermo Jarrel ASH, modelo Spectrometer Polyscan $^{\mathrm{TM}}$ 61E. Se emplearon longitudes de onda de 317.933 nm para el calcio y $178.287 \mathrm{~nm}$ para el fósforo.

\subsubsection{DIFRACCIÓN DE RAYOS-X}

La identificación de las fases cristalinas presentes tanto en la HA como en los materiales con Mg-PSZ se realizó mediante un difractómetro de polvo automático SIEMENS D-500 con radiación $\mathrm{Cu} \mathrm{K} \alpha$.

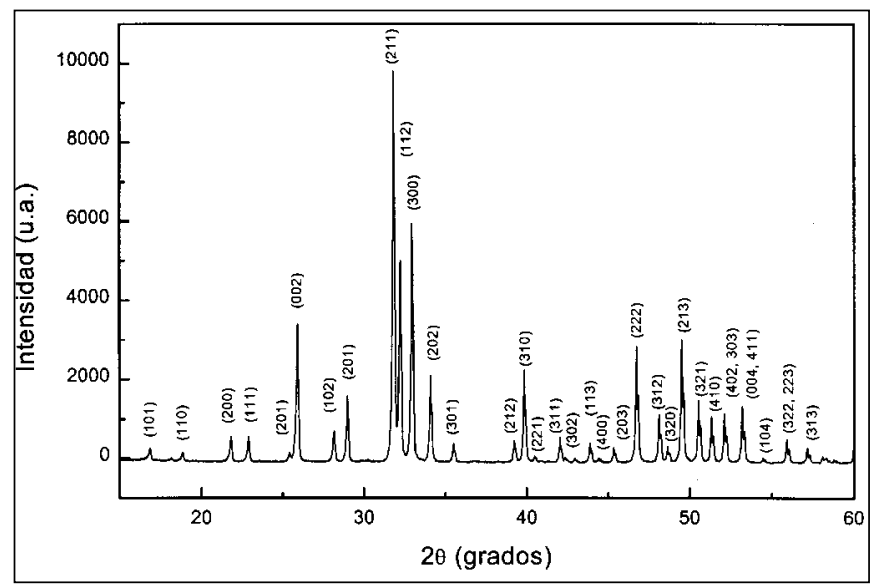

Fig. 1. Difractograma de la HA sinterizada a $1350^{\circ} \mathrm{C}$ durante 4 horas en atmósfera de oxígeno húmedo.

\subsubsection{MICROSCOPÍA ELECTRÓNICA DE BARRIDO (MEB) Y MICROANÁLISIS POR MICROSONDA ELECTRÓNICA.}

Para su observación los materiales fueron embebidos en resina, las superficies pulidas y recubiertas con una fina película de oro. Se empleó un MEB HITACHI S-2300 con sistema de imágenes digitales acoplado. Se trabajó a una tensión de aceleración de $20 \mathrm{kV}$. La determinación del tamaño de grano se realizó mediante técnicas de análisis de imágenes. En el análisis cuantitativo por microsonda electrónica se utilizó un equipo tipo Castaing CAMECA modelo CAMEBAX SX 50 con cuatro espectrómetros verticales WDS de rayos-X de separación por longitudes de onda (Wavelength Dispersive System). Las experiencias se realizaron con una tensión de aceleración de 20 $\mathrm{kV}$ y una corriente de sonda de $10 \mathrm{nA}$.

\subsubsection{ESPECTROSCOPÍA INFRARROJA (FTIR)}

Para la realización de los espectros infrarrojos los materiales se prepararon en pastillas con $\mathrm{KBr}$. Los registros se llevaron a cabo en un intervalo de $500-4000 \mathrm{~cm}^{-1}$ en un Espectrómetro Infrarrojo con Transformada de Fourier (FTIR) BOMEM MB120 con detector DTGS.

\subsubsection{CARACTERIZACION MECÁNICA}

Para determinar la densificación de las cerámicas las dimensiones de las piezas se midieron con un micrómetro mientras que el peso se determinó con una balanza analítica. La densidad de los materiales HA-PSZ se calculó considerando, 3.16 $\mathrm{g} / \mathrm{cm}^{3}$ para la HA pura y $3.47 \mathrm{~g} / \mathrm{cm}^{3}$ para el material compuesto por $80 \%$ en peso de HA y $20 \%$ en peso de Mg-PSZ. En la caracterización mecánica, se empleó un microdurómetro Vickers (Carl Zeiss). Previamente los materiales fueron incluidos en resina y la superficie de trabajo fue pulida. La carga de $1.96 \mathrm{~N}$ se mantuvo por $15 \mathrm{~s}$ y la tenacidad a la fractura $\left(\mathrm{K}_{1 \mathrm{C}}\right)$ se calculó empleando la ecuación (9):

$$
0.0495^{*}\left(\mathrm{H}_{\mathrm{v}} \mathrm{a}^{1 / 2}\right)^{*}(\mathrm{E} / \mathrm{H})^{2 / 5 *}(\mathrm{c} / \mathrm{a})^{[(\mathrm{c} / 18 \mathrm{a})-1.51]}
$$

donde $\mathrm{H}_{\mathrm{v}}$ es la dureza Vickers, a es la media diagonal de la indentación Vickers y c es la longitud de la grieta que provoca

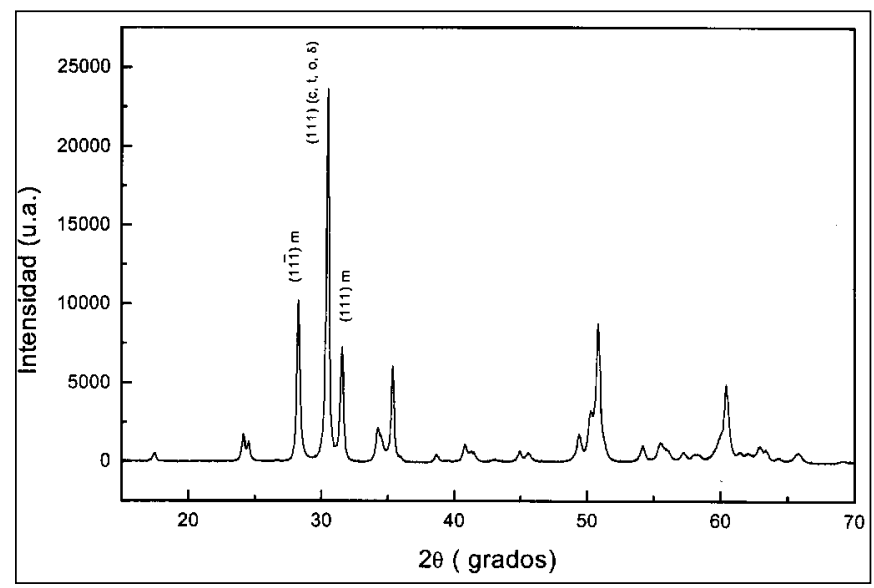

Fig. 2. Difratograma de circona estabilizada con magnesia (Mg-PSZ) sinterizada a $1450{ }^{\circ} \mathrm{C}$ durante 4 horas. 


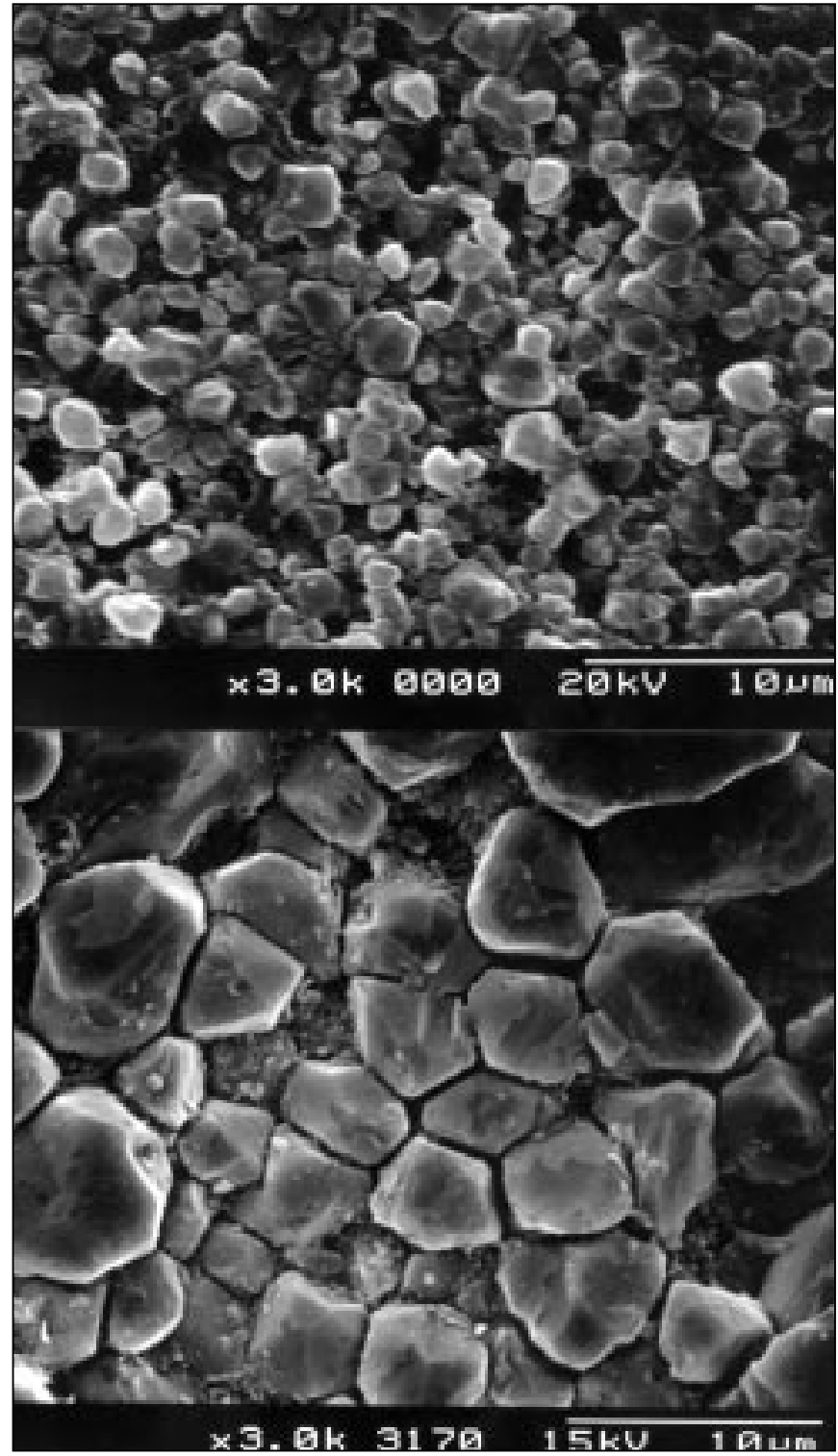

Fig. 3a y 3 b.Influencia de la temperatura de sinterización.

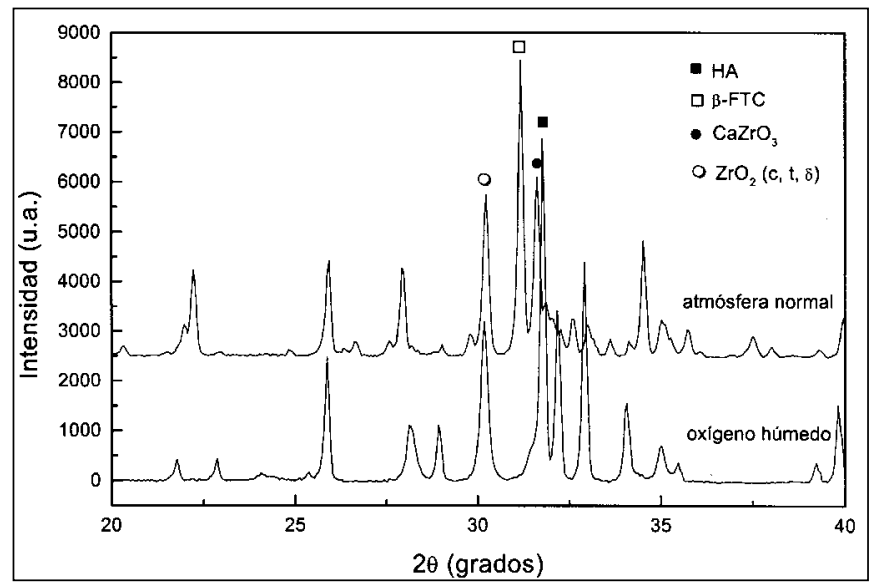

Fig. 4. Difractograma de muestras de HA-MgPSZ sinterizadas en aire y en oxígeno húmedo a $1250{ }^{\circ} \mathrm{C}$ durante 4 horas. la indentación. El módulo de Young (E) se calculó de acuerdo con la formula de Evans (10) empleando $1.96 \mathrm{~N}$ durante $15 \mathrm{~s}$.

$$
\mathrm{b} / \mathrm{a}=0.142-0.45(\mathrm{H} / \mathrm{E})
$$

donde b y a son respectivamente las diagonales cortas y largas del indentador Knoop.

\section{RESULTADOS}

\subsection{Caracterización de la HA y la Mg-PSZ}

El análisis químico de la $\mathrm{HA}$ calcinada a $700^{\circ} \mathrm{C}$ durante 2 horas reveló una relación molar calcio/fósforo de $1.69 \pm 0.01$ muy cercana al valor de 1.67 correspondiente a una hidroxiapatita estequiométrica. El difractograma de polvo muestra una única fase cristalina identificada como hidroxiapatita, además no hay evidencia de descomposición térmica cuando el material se calentó a $1350^{\circ} \mathrm{C}$ durante 4 horas (Figura 1).

El difractograma de la Mg-PSZ (Figura 2) presenta los picos característicos de las tres fases que componen las circonas parcialmente estabilizadas (11). La fracción en peso de fase monoclínica se determinó según la fórmula propuesta por Batchelor et al.(12):

$$
\begin{gathered}
W_{m}=\frac{P X_{m}}{1+(P-1) X_{m}} \\
X_{m}=\frac{\left(1+\frac{1}{0.68}\right) I_{m}^{111}}{I_{t+c+o+\delta}^{111}+\left(1+\frac{1}{0.68}\right) I_{m}^{111}} \\
W_{m}=0.41 \pm 0.03
\end{gathered}
$$

donde los subíndices $m, t, c, o$ y $\delta$ se refieren a las fases monoclínica, tetragonal, cúbica, ortorrómbica y delta respectivamente. El término $\mathrm{I}(h k l)$ representa la intensidad integrada de la linea de difracción indicada por sus índices de Miller. El término 0.68 se obtiene a partir de la relación entre la intensidad de los picos $111_{\mathrm{m}}$ y $1 / 1_{\mathrm{m}}$ de la circona monoclínica. El término $\mathrm{P} \approx 1.1$ para la mezcla de fases que se encuentran normalmente en la Mg-PSZ (13).

Algunos autores (14), trabajando con piezas monolíticas de Mg-PSZ, emplean los picos $\{400\}$ para discriminar entre las fases tetragonal y cúbica pero esto es prácticamente imposible cuando se trabaja con muestras que han sido trituradas pues dichos picos presentan muy poca intensidad debido a la transformación de parte de la fase tetragonal en fase monoclínica por el efecto de la molienda (15).

El estudio por Microscopía Electrónica de Barrido permitió apreciar la diferencia en el crecimiento de grano entre las muestras sinterizadas a $1250^{\circ} \mathrm{C}$ y a $1350^{\circ} \mathrm{C}$. El tamaño promedio de grano para las muestras de $1250^{\circ} \mathrm{C}$ fue de $1.55 \pm 0.6 \mu \mathrm{m}$ mientras que para aquellas tratadas a $1350^{\circ} \mathrm{C}$ fue de $6,5 \pm 4,5$ mm (Figuras 3a y 3b). 


\subsection{Caracterización de los cerámicos compuestos de HA-} PSZ.

\subsubsection{DIFRACCIÓN DE RAYOS-X.}

Los resultados de la difracción de rayos- $X$ revelaron que durante la sinterización en atmósfera húmeda, la hidroxiapatita se mantuvo como la fase mayoritaria a pesar de la presencia de circona. Este comportamiento resultó ser muy diferente al que experimentó dicho material al ser sinterizado en aire donde es evidente la formación de zirconato cálcico (CZ) producto de la reacción entre la PSZ y el óxido de calcio liberado en la descomposición de la HA (Figura 4). Además, se observó la presencia del $\beta$-fosfato tricálcico ( $\beta$-TCP) como fase mayoritaria, producto de la descomposición de la HA.

\subsubsection{ESPECTROSCOPÍA INFRARROJA}

Esta técnica se empleó para confirmar la estabilidad de la fase de hidroxiapatita en presencia de las partículas de $\mathrm{Mg}$ PSZ. En los espectros de las muestras sinterizadas en oxígeno húmedo se pueden apreciar las bandas de absorción características de los principales grupos funcionales que componen la HA, estas bandas aparecen a los 3568, 1090, 1043, 961, 601 y $570 \mathrm{~cm}^{-1}$. Por el contrario en los registros de las piezas sinterizadas a la misma temperatura y tiempo pero en aire aparecen tres nuevas bandas a los 1119, 969 y $944 \mathrm{~cm}^{-1}$ correspondientes al grupo $\mathrm{PO}_{4}^{3-}$ del $\beta$-TCP (Figura 5).

\subsubsection{MICROSCOPÍA ELECTRÓNICA DE BARRIDO Y MICROSONDA ELECTRÓNICA.}

La formación del $\mathrm{CZ}$ producto de la difusión del calcio desde la HA hacia la partícula de Mg-PSZ se pone de manifiesto cuando se realizaron observaciones con el MEB. Específicamente cuando se emplea imágenes de electrones retrodifundidos, la zona de la interfaz entre las partículas de Mg-PSZ y la matriz de HA presenta un color intermedio entre el color oscuro de la matriz (rica en calcio) y el color claro brillante de la partícula de Mg-PSZ rica en zirconio. El análisis cuantitativo a través de la Microsonda Electrónica reveló la presencia de zirconato cálcico estequiométrico $\left(\mathrm{CaZrO}_{3}\right)$.

\subsubsection{DETERMINACIÓN DE DENSIDADES Y PROPIEDA- DES MECÁNICAS.}

Las densidades y las propiedades mecánicas obtenidas para los materiales sinterizados a $1250^{\circ} \mathrm{C}$ durante 4 horas se muestran en la tabla I. Los valores de densidades, $\mathrm{H}$, E y $\mathrm{K}_{1 \mathrm{C}}$ para la HA corresponden al material obtenido a una presión de compactación de $440 \mathrm{MPa}$. En el caso de la HA la densidad obtenida es superior al $98 \%$ mientras que para los materiales que contienen Mg-PSZ los valores de esta propiedad están cercanos al $95 \%$. Como se puede apreciar los valores de la tenacidad a la fractura $\left(\mathrm{K}_{1 \mathrm{C}}\right)$ aumentan en el caso de los materiales que contienen Mg-PSZ hasta alcanzar un valor que prácticamente duplica al obtenido para la HA pura como es el caso de

TABLA I.

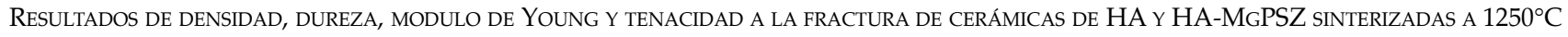
DURANTE 4 HORAS EN OXÍGENO HÚMEDO.

\begin{tabular}{|c|c|c|c|c|}
\hline Material & Densidad $(\%)$ & H (GPa) & E (GPa) & $K_{1 C}\left(M a^{1 / 2}\right)$ \\
\hline $\mathrm{HA}(440)^{*}$ & $96.27 \pm 0.43$ & $4.14 \pm 0.28$ & $213 \pm 26$ & $0.81 \pm 0.07$ \\
\hline HA-MgPSZ (264)* & $92.22 \pm 0.66$ & $2.89 \pm 0.19$ & $103 \pm 19$ & $1.62 \pm 0.12$ \\
\hline HA-MgPSZ (306)* & $92.94 \pm 0.47$ & $2.56 \pm 0.22$ & $114 \pm 16$ & $1.66 \pm 0.10$ \\
\hline HA-MgPSZ (352)* & $93.95 \pm 0.39$ & $2.77 \pm 0.14$ & $139 \pm 25$ & $1.93 \pm 0.12$ \\
\hline HA-MgPSZ (440)* & $94.35 \pm 0.52$ & $2.94 \pm 0.20$ & $112 \pm 17$ & $1.84 \pm 0.09$ \\
\hline
\end{tabular}

* Presión de compactación en $\mathrm{MPa}$

TABLA II.

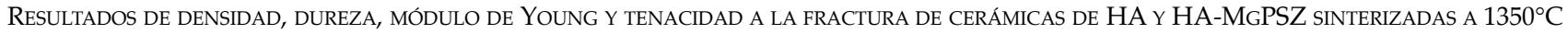
DURANTE 4 HORAS EN OXÍGENO HÚMEDO.

\begin{tabular}{|c|c|c|c|c|}
\hline Material & Densidad (\%) & H (GPa) & E (GPa) & $\mathrm{K}_{1 \mathrm{C}}\left(\mathrm{MPa} \mathrm{\textrm {m } ^ { 1 / 2 } )}\right.$ \\
\hline HA $(440)^{*}$ & $97.90 \pm 0.63$ & $3.28 \pm 0.42$ & $206 \pm 48$ & $1.06 \pm 0.15$ \\
\hline HA-MgPSZ $(264)^{*}$ & $94.31 \pm 0.48$ & $3.54 \pm 0.22$ & $151 \pm 40$ & $1.68 \pm 0.17$ \\
\hline HA-MgPSZ (306)* & $92.63 \pm 0.53$ & $3.53 \pm 0.34$ & $102 \pm 31$ & $1.42 \pm 0.16$ \\
\hline HA-MgPSZ $(352)^{*}$ & $94.08 \pm 0.60$ & $3.38 \pm 0.23$ & $120 \pm 24$ & $1.38 \pm 0.07$ \\
\hline HA-MgPSZ $(440)^{*}$ & $93.96 \pm 0.55$ & $3.56 \pm 0.21$ & $144 \pm 25$ & $1.69 \pm 0.14$ \\
\hline
\end{tabular}

* Presión de compactación en $\mathrm{MPa}$ 


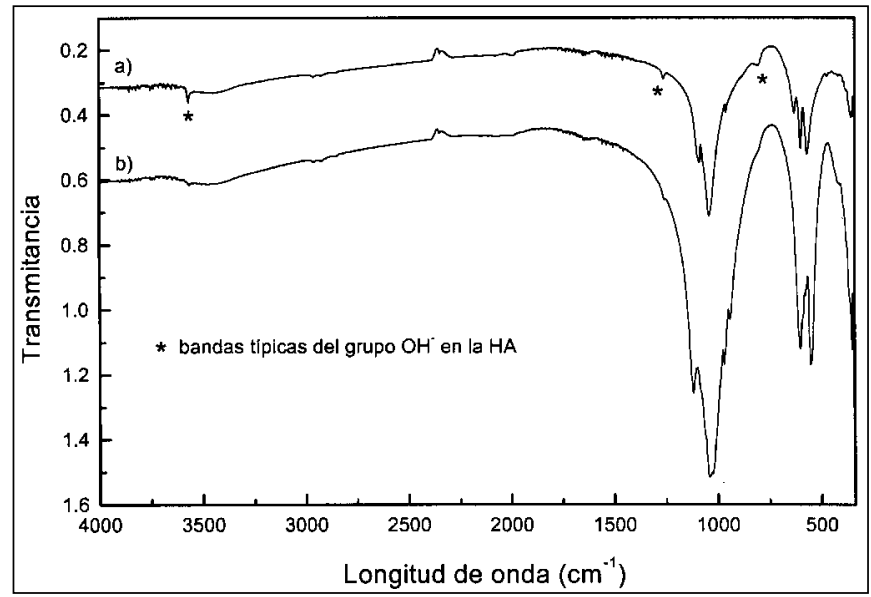

Fig. 5. Espectros infrarrojos de muestras de HA-MgPSZ sinterizadas a) en oxígeno húmedo correspondiendo el patrón de infrarrojo a una HA bien cristalizada, b) en aire, el patrón corresponde a un $\beta$-FTC donde se observan la ausencia de las bandas de los grupos $\mathrm{OH}^{-}$que componen la HA.

$1.93 \pm 0.12\left(\mathrm{MPa} \mathrm{m}^{1 / 2}\right)$ que se alcanzó para las muestras prensadas a $352 \mathrm{MPa}$ y sinterizadas a $1250^{\circ} \mathrm{C}$ durante 4 horas. Por otra parte, en el caso de $\mathrm{H}$ y E los valores para la HA siempre fueron mayores que los obtenidos para los materiales que contienen Mg-PSZ.

La tabla II muestra los valores obtenidos para los materiales sinterizados a $1350^{\circ} \mathrm{C}$ durante 4 horas. A esta temperatura los valores de la $\mathrm{K}_{1 \mathrm{C}}$ son también mayores que los de la HA aunque no se observa la misma tendencia a aumentar con la presión de compactación y no se alcanzan valores tan altos como en el caso de las muestras sinterizadas a $1250^{\circ} \mathrm{C}$.

\section{DISCUSIÓN}

La estabilidad de la fase apatítica en los materiales que contenían Mg-PSZ, cuando las sinterizaciones se llevaron a cabo en atmósfera de oxígeno húmedo, ha quedado demostrada a través de los resultados de la difracción de rayos- $X$ y la espectroscopía infrarroja. Incluso para temperaturas altas como es el caso de $1350^{\circ} \mathrm{C}$ y tiempos de sinterización de 4 horas es la HA la única fase fosfatocálcica presente. Se debe destacar que no aparece ningún pico asociado a la formación de $\mathrm{CZ}$, todo lo contrario a lo que sucede para las muestras sinterizadas en aire donde es el CZ la fase mayoritaria.

Los espectros infrarrojos de las muestras sinterizadas en oxígeno húmedo permiten corroborar lo obtenido por difracción de rayos- $X$, se aprecian las bandas asociadas a los grupos funcionales que componen la HA, específicamente la banda correspondiente a la vibración de valencia fundamental del grupo hidroxilo $\left(\mathrm{OH}^{-}\right)$estructural de la $\mathrm{HA}$, además el espectro IR muestra una buena coincidencia en la posición de las demás bandas características de la HA. En el caso de las muestras sinterizadas en aire han desaparecido las bandas correspondientes al grupo hidroxilo y aparecen bandas típicas del $\beta$ FTC.

El efecto de la influencia de la atmósfera de oxígeno húmedo sobre la estabilidad térmica de la HA lo podemos explicar partiendo de que la descomposición de la HA a temperaturas elevadas está asociado con dos procesos el primero de ellos es el denominado de deshidroxilación, es decir la pérdida progresiva de radicales $\mathrm{OH}^{-}$con el aumento de la temperatura. Dicho proceso se puede describir de acuerdo con la siguiente reacción:

$$
\mathrm{Ca}_{10}\left(\mathrm{PO}_{4}\right)(\mathrm{OH})_{2} \rightarrow \mathrm{Ca}_{10}\left(\mathrm{PO}_{4}\right)(\mathrm{OH})_{2-2 \mathrm{x}} \mathrm{O}_{\mathrm{x}} \Delta_{\mathrm{x}}+\mathrm{x} \mathrm{H}_{2} \mathrm{O} \uparrow
$$

El producto deficiente en iones hidroxilo $\mathrm{Ca}_{10}\left(\mathrm{PO}_{4}\right)(\mathrm{OH})_{2}$. ${ }_{2 x} \mathrm{O}_{x} \Delta_{x}(\Delta=$ vacante, $\mathrm{x}<1)$ se conoce como oxihidroxiapatita $\mathrm{y}$ su existencia ha sido identificada por difracción de rayos- $X$ y estudios de espectroscopía infrarroja (16). En el caso que nos ocupa la humedad existente en la atmósfera de sinterización mantiene el equilibrio de la reacción de descomposición desplazado hacia la HA con lo cual se garantiza su estabilidad. El segundo proceso de descomposición de la HA es la descomposición propiamente dicha de la HA con la formación de FTC y el fosfato tetracálcico (TetrCP):

$$
\mathrm{Ca}_{10}\left(\mathrm{PO}_{4}\right)(\mathrm{OH})_{2-2 \mathrm{x}} \mathrm{O}_{\mathrm{x}} \Delta_{\mathrm{x}} \rightarrow 2 \mathrm{Ca}_{3}\left(\mathrm{PO}_{4}\right)_{2}+\mathrm{Ca}_{4} \mathrm{P}_{2} \mathrm{O}_{9}+\left(\mathrm{H}_{2} \mathrm{O}\right)_{1-\mathrm{x}}
$$

Algo muy similar a lo descrito anteriormente es lo que sucede al sinterizar muestras de HA que contienen Mg-PSZ en aire. En nuestro caso, debido a las temperaturas de trabajo, no hemos detectado la presencia del TetrCP

La efectividad del proceso de sinterización en estas condiciones ha quedado demostrada en los altos grados de densificación obtenidos así como con el crecimiento de grano de la HA tal y como se aprecia en las microfotografías de MEB. Los valores de densidad de los materiales con Mg-PSZ también se pueden considerar como buenos teniendo en cuenta hasta el presente materiales de estas características solo se habían obtenido empleando prensado isostático en caliente (HIP) y como agente de refuerzo partículas de Y-PSZ, en estos casos los valores de densidad obtenidos están alrededor del $97 \%(5,17)$.

El aumento de la tenacidad a la fractura en los materiales que contienen $\mathrm{Mg}$-PSZ respecto a la HA pura es un indicador de que las partículas de PSZ pueden actuar como agente de refuerzo cuando se encuentran en una matriz de HA. La expli-

TABLA III.

COMPARACIÓN DE LOS VALORES DE TENACIDAD A LA FRACTURA CON OTROS REPORTADOS PARA MATERIALES SIMILARES.

\begin{tabular}{|c|c|c|}
\hline Material & Método & $\mathbf{K}_{\mathbf{1 C}}\left(\mathbf{M P a} \mathbf{~ m}^{\mathbf{1} / \mathbf{2}}\right)$ \\
\hline HA-MgPSZ $(20 \%$ en peso) & $1.93 \pm 0.12$ \\
\hline HA-YPSZ (20\% en peso) [17] & Prensado uniaxial & 2.3 \\
\hline HA-YPSZ (26.8\% en peso) [5] & Prensado isostático en caliente & 2.1 \\
\hline
\end{tabular}

* Material sinterizado a $1250^{\circ} \mathrm{C}$ durante 4 horas en oxígeno húmedo 
cación de este comportamiento se explica, como hemos referido con anterioridad, por la capacidad de las partículas de PSZ de impedir la propagación de grietas en el material sometido a esfuerzos.

Para los materiales que contienen Mg-PSZ sinterizados a $1350^{\circ} \mathrm{C}$ durante 4 horas en atmósfera húmeda, se observa un aumento de la dureza comparado con los valores obtenidos a $1250^{\circ} \mathrm{C}$. Los demás parámetros presentan un comportamiento similar al obtenido para las muestras sinterizadas a $1250^{\circ} \mathrm{C}$, si bien los valores de $\mathrm{K}_{1 \mathrm{C}}$ son algo inferiores. El aumento de la dureza puede provocar un ligero aumento de la fragilidad de estos materiales que se manifiesta en una cierta disminución de los valores de la $\mathrm{K}_{1 \mathrm{C}}$.

En la tabla III, se compara el valor de la $\mathrm{K}_{1 \mathrm{C}}$ más alto obtenido para los materiales que contienen Mg-PSZ con los valores reportados por autores que emplean Y-PSZ como agente de refuerzo y prensado isostático en caliente $(5,17)$.

Como conclusión, se puede afirmar que es posible reforzar las cerámicas de HA empleando como agente de refuerzo partículas de Mg-PSZ y utilizando prensado uniaxial y sinterizaciones en atmósfera húmeda. Se obtienen materiales más tenaces, con lo que se puede ampliar el campo de aplicación de las cerámicas de HA, si bien esto son solo resultados preliminares quedando pendientes otras pruebas mecánicas que contribuirán a ampliar lo expuesto en este trabajo.

\section{AGRADECIMIENTOS}

Los autores agradecen al programa de becas Mutis de la Agencia Española de Cooperación Internacional por la ayuda brindada a través de una beca pre-doctoral a Delgado GarcíaMenocal. También manifestamos nuestro agradecimiento al Centro de Servicios Científico-Técnicos de la Universidad de Barcelona por la colaboración técnica.

\section{BIBLIOGRAFÍA}

1. K. de Groot, "Bioceramics of Calcium Phosphate”, pp. 1-32, CRC Press, Boca Raton, Fl, 1983.

2. K. de Groot and R. Le Geros, "Significance of Porosity and Physical Chemistry of Calcium Phosphate Ceramics", pp. 268-77 en Bioceramics: Material Characteristics Versus in Vivo Behavior, Vol. 523. Ed. P. Ducheyne and J. Lemons. Annals of New York Academy of Science, New York, 1988.
3. D. F. Williams, "The Biocompatibility and Clinical Uses of Calcium Phosphate Ceramics", pp. 43-66 en Biocompatibility of Tissue Analogs, Vol. II. Ed. D. F. Williams. CRC Press, Boca Raton, FL, 1985.

4. K. de Groot, C. de Putter, P. Smitt, A. Driessen, “Mechanical failure of artificial teeth made of dense calciumhydroxyapatite", Sci. Ceram. 11, 433-437 (1981).

5. N. Takaqi, M. Mochida, N. Uchida, K. Saito, K. Uematsu, “Filter cake forming and hot isostatic pressing for TZP-dispersive hydroxyapatite composite", J. Mater. Sci.: Mater. Med. 3, 199-203 (1992).

6. D. L. Porter and A. H. Heuer, "Mechanisms of Toughening Partially Stabilized Zirconia (PSZ)", J. Am. Ceram. Soc. 60 [3-4], 183-84 (1977).

7. J. Caetano-Zurita, O. Bermúdez, I. López-Valero, E. B. Stucchi, J. A. Varela, J. A. Planell and S. Martínez, "Mechanical Behaviour of a HydroxyapatiteZirconia Particles Composite”, pp. 267-271 en Bioceramics, Volume 7, Ed. Ö. H. Anderson and Yli-Urpo. (Proceeding of the $7^{\text {th }}$ International Symposium on Ceramics in Medicine, Turku, Finland, July 1994), ButterwothHeinemann Ltd., 1994.

8. E. Pauchiu, T. Wang, K. Chaki, "Sintering behaviour and mechanical properties of hydroxyapatite and dicalcium phosphate", J. Mater. Sci.: Mater. Med. 4, 150-158 (1993).

9. K. M. Liang, G. Orange, G. Fantozzi, “Evaluation by indentation of fracture toughness of ceramic materials", J. Mater. Sci. 25, 207-214 (1990).

10. D. B. Marshall, T. Noma, A. G. Evans, "A simple method for determining elastic-modulus-to-hardness ratios using Knoop indentation measurements", J. Am. Ceram. Soc. 65, C-175-C-176 (1982).

11. M. -O. Guillou, J. L. Henshall, R. M. Hooper, "The measurement of surface contact fatigue and its application to engineering ceramics", Mat. Sci. Eng. A209, 116-127 (1996).

12. W. J. Batchelor, T. R. Finlayson, and G. L. Kelly, "Monoclinic Phase Measurement in Mg-PSZ using X-ray difracction", J. Am. Ceram. Soc. 79 [9] 2477-80 (1996).

13. C. J. Howard and E. H. Kisi, "'Polymorph Method'Determination of Monoclinic Zirconia in Partially Stabilized Zirconia Ceramics", J. Am. Ceram. Soc. 73 3096-99 (1990).

14. N. Iwamoto, N. Umesaki and S. Endo, "Structural Investigation of PlasmaSpayed Zirconia coatings by Raman-Spectroscopy", Yogyo Kyokai-Shi 93, 281-288 (1985)

15. R. J. Hill, B. E. Reichert, “Measurement of Phase Abundance in MagnesiaPartially-Stabilized Zirconia by Rietveld Analysis of X-ray Diffraction Data", J. Am. Ceram. Soc. 73 [10] 2822-27 (1990).

16. T. Kijima, M. Tsutsumi, "Preparation and Thermal Properties of Dense Polycristalline Oxyhydroxyapatite", J. Am. Ceram. Soc. 62 [9-10] 455-460 (1978).

17. K. Ioku, M. Yoshimura and S. Somiya, "Microstructure and mechanical properties of hydroxyapatite ceramics with zirconia dispersion prepared by post-sintering", Biomaterials, 11 57-61 (1990). 